\title{
On the Dynamics of Transition of a Classical System to Equilibrium State
}

\author{
Sami M. AL-Jaber \\ Department of Physics, An-Najah National University, Nablus, Palestine \\ Email:Jaber@najah.edu
}

How to cite this paper: AL-Jaber, S.M (2018) On the Dynamics of Transition of a Classical System to Equilibrium State. Applied Mathematics, 9, 897-906. https://doi.org/10.4236/am.2018.98062

Received: July 30, 2018

Accepted: August 19, 2018

Published: August 22, 2018

Copyright (C) 2018 by author and Scientific Research Publishing Inc. This work is licensed under the Creative Commons Attribution International License (CC BY 4.0).

http://creativecommons.org/licenses/by/4.0/

\begin{abstract}
In this work we consider a spring with one end is fixed and the other is connected to a block of mass $M$ located on a horizontal rough table. The other side of the block is connected to a massless rope that passes over a frictionless pulley at the end of the table and a second block of mass $m$ is hanged at the rope's other end. For this system, we analyze and discuss its dynamics of motion as function of time when the second block is released. In particular, the displacement of the system at the end of each half-cycle of motion, the total distance, and the work done against friction are derived. An interesting result is obtained for the case when the table is frictionless. It is found that there is still a work done by friction whose magnitude is exactly the same as the stored energy in the spring.
\end{abstract}

\section{Keywords}

Classical Oscillating System, Spring-Mass System with Friction, Energy Dissipation

\section{Introduction}

The problem of the transition of a physical system from a non-equilibrium state to a final permanent equilibrium state plays a central role in understanding the dynamics of the behavior of the system during this transition [1]-[8]. In such systems, energy dissipation or energy transfer is a crucial quantity for the system to be able to undergo a transition from non-equilibrium to final equilibrium state [9] [10] [11] [12] [13]. A well-known system which demonstrates the role and mechanisms of energy dissipation during its transition from a non-equilibrium to an equilibrium state is the two-capacitor problem which has been under investigation by many authors [14]-[21]. Classical systems, which involve energy dissipation in their transition from non-equilibrium to equilibrium state, have re- 
cently attracted much attention of many authors [22] [23] [24] [25] [26]. Students usually have difficulty in understanding the dynamics involved in such systems during their transition from non-equilibrium to equilibrium state [27] and some activities and models have been proposed to overcome some of their main difficulties [28] [29]. A major difficulty for students arises when they deal with final equilibrium state for a spring-mass system. To be specific, we consider the following spring-mass system: A block of mass $M$, rests on a horizontal table, is attached to one end of a spring whose other end is fixed to a vertical fixed wall. The other side of the block is attached to a massless rope that passes over a frictionless pulley and another block of mass $m$ is attached to the other end of the rope and hanged vertically off the table. The aim of this paper is to study and analyze the dynamics and the behavior of this system during its transition to final stable equilibrium state after the release of the hanged mass $m$. In this paper, we apply the Lagrangian method and solve Lagrange equations to determine the position of the hanged mass at any half-cycle $n$. Our results show the suitable $n$ which is needed for the system to attain its final equilibrium state. It is also shown that one can get the total distance covered by the system. In addition, the dissipated energy in the process is analyzed and its relation to the energy stored in the spring is pointed out. It is remarkable to note that in the limit when the coefficient of friction goes to zero, our results show that the energy dissipated is exactly the same as the energy stored in the spring and so each is half the initial gravitational potential of the hanged mass.

\section{Spring-Mass System and Equation of Motion}

Our system consists of a spring (spring's constant $k$ ), on a horizontal table with coefficient of kinetic friction $\mu$, which is fixed at one end and the other end is attached to a block of mass $M$ which is connected to a massless rope that passes over a frictionless pulley fixed (but free to rotate) at the end of the table and another block of mass $m$ attached to the rope and hanged off the table (see Figure 1 below).

Initially, the system is at rest and the zero reference is set at the position of $m$. After the system is released, the maximum downward distance, $x_{0}$ covered by $m$ occurs when it comes momentarily to rest and this can be determined by requiring that the work done by frictional force is equal to the change in the mechanical energy, with the result;

$$
x_{0}=\frac{2}{k}(m g-\mu M g) .
$$

This occurs when $m$ reaches zero velocity and $M$ reaches maximum displacement to the right. In the second step $m$ returns up and $M$ moves to left until the system gets to zero velocity after which $M$ moves to right and $m$ moves downward and the system repeats the motion with decreasing amplitude due to the work done by frictional force between $M$ and the table. Finally the system comes to final stable equilibrium state and the final displacement $x_{f}$ made by the system is determined by balance of forces with the result; 


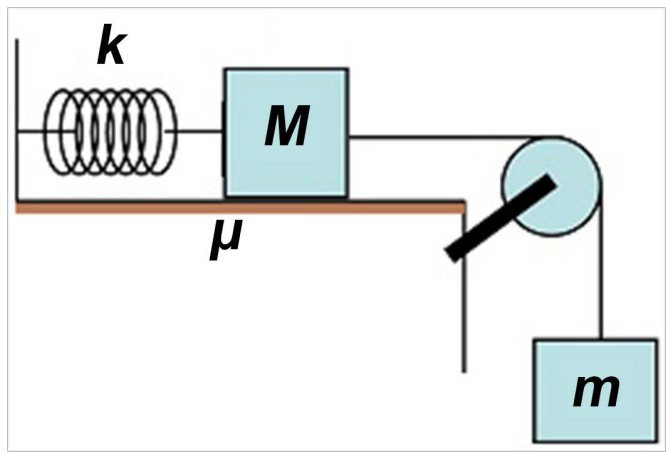

Figure 1. Two masses connected to a spring.

$$
x_{f}=\frac{1}{k}(m g-\mu M g),
$$

which is exactly half $x_{0}$. We must note that initially (just before the release) the system is in a non-equilibrium state and finally in an equilibrium state. The dynamics which controls the behavior of the system between these two states is undermined and the physics involved is not utilized. Our aim in this paper is to examine the behavior of the system during its transition between these two states. Specifically, we will find the position of $m$ during any given half cycle $n$, its final position at the end of each half cycle and the number of half cycles made by the system before it comes to the final equilibrium state.

Let $x$ be a generalized coordinate, the Lagrangian of the system is

$$
L=\frac{1}{2}(m+M) \dot{x}^{2}-\left(\frac{1}{2} k x^{2}-m g x\right),
$$

where $x$ and $\dot{x}$ are the position and speed of $m$. The Lagrange's equation of motion for the generalized coordinate $x$ reads

$$
\frac{\mathrm{d}}{\mathrm{d} t}\left(\frac{\partial L}{\partial \dot{x}}\right)-\frac{\partial L}{\partial x}=Q
$$

where $Q$ is the frictional force between the surface and $M$ and is given by

$$
Q=(-1)^{n} \mu M g
$$

with $n$ is the number of half-cycle of the motion. For odd $n, M$ is moving to the right so that the frictional force is negative, while for even $n, M$ is moving to the left so that the frictional force is positive. Inserting Equations (4) and (5) into Equation (3) gives

$$
\ddot{x}+\omega^{2} x=\frac{1}{m+M}\left(m g+(-1)^{n} \mu M g\right),
$$

where $\omega^{2}=k /(m+M)$. Equation (6) is an inhomogenous first order differential equation, whose solution after applying the initial condition $\dot{x}_{n}=0$ for each half-cycle, is

$$
x_{n}=A_{n} \cos (\omega t)+\frac{1}{k}\left(m g+(-1)^{n} \mu M g\right) .
$$


The constant $A_{n}$ can be found as follows:

For the first half cycle, $(0, \pi)$, i.e. $n=1$, we have $x_{1}(0)=0$ so that equation (7) gives $A_{1}=\frac{1}{k}(-m g+\mu M g)$. For the second half cycle, $(\pi, 2 \pi)$, i.e. $n=2$, we require that the final position of $m$ at end of the first half cycle equals the initial position at the beginning of the second half cycle, which means the $x_{1}(\pi)=x_{2}(\pi)$. This gives, $A_{2}=\frac{1}{k}=(-m g+3 \mu M g)$. In a similar way, for the $n^{t h}$ half cycle, $((n-1) \pi, n \pi)$, we require the position of $m$ at end of the $(n-1)$ half cycle equals to its position at the beginning of the $n^{\text {th }}$ half cycle, which means $x_{n-1}((n-1) \pi)=x_{n}((n-1) \pi)$. One might expect that

$$
A_{n}=\frac{1}{k}(-m g+(2 n-1) \mu M g)
$$

$d$ hence Equation (7) becomes

$$
x_{n}=\frac{1}{k}(-m g+(2 n-1) \mu M g) \cos \omega t+\frac{1}{k}\left(m g+(-1)^{n} \mu M g\right),
$$

which we prove by mathematical induction as follows: For $n=1$, we already derived $A_{1}$ which upon its substitution into Equation (7) gives $x_{1}$. We assume Equation (9) is true for any $n$, and we need to show that it is true for $n+1$. For the $(n+1)^{\text {th }}$ half cycle, $(n \pi,(n+1) \pi)$, we require that $x_{n}(n \pi)=x_{n+1}(n \pi)$. This means that, at $\omega t=n \pi$, the position of $m$ at the end on the $n^{\text {th }}$ half cycle is equal to its position at the beginning of the $(n+1)^{\text {th }}$ half cycle. Using Equations (7) and (9), we get

$$
\begin{aligned}
& \frac{g}{k}(-m+(2 n-1) \mu M) \cos (n \pi)+\frac{g}{k}\left(m+(-1)^{n} \mu M\right) \\
& =A_{n+1} \cos (n \pi)+\frac{g}{k}\left(m+(-1)^{n} \mu M\right)
\end{aligned}
$$

The above equation immediately yields, for both case $n=$ odd and $n=$ even, $A_{n+1}$, namely

$$
A_{n+1}=\frac{1}{k}(-m g+(2 n+1) \mu M g),
$$

which, upon its substitution into Equation (7), gives us

$$
x_{n+1}=\frac{1}{k}(-m g+(2 n+1) \mu M g) \cos (\omega t)+\frac{1}{k}\left(m g+(-1)^{n+1} \mu M g\right) .
$$

This is exactly $x_{n}$ given in Equation (9) with $n \rightarrow n+1$, and this completes the proof.

Equation (9) yields for $n=$ odd, with $\omega t=n \pi$

$$
x_{n}=\frac{2}{k}(m g-n \mu M g)
$$

which gives the lowest position of $m$ and the maximum right displacement of $M$ when they come momentarily to rest at the end of the $n^{\text {th }}$ half-odd cycle. For $n=$ 
even, Equation (9) yields,

$$
x_{n}=\frac{2 n}{k} \mu M g,
$$

which gives the highest position of $m$ and the maximum left displacement of $M$ when they come momentarily to rest at the end of the $n^{\text {th }}$ half-even cycle.

\section{The Final Equilibrium State}

Now the question is where the system comes to equilibrium permanently? We claim that this occurs at a final position given by

$$
X_{f}=\frac{1}{2}\left(x_{n}+x_{n+1}\right) \text {. }
$$

Using Equation (9) for $x_{n}$, Equation (14) gives,

$$
X_{f}=\frac{(m g \pm \mu M g)}{k},
$$

where the signs $+(-)$ are for $n=$ odd (even). The result given in Equation (15) is expected, since for the $n=$ odd case the motion is from odd $n$ to even $(n+1)$ which means that mass $m$ is moving upward and $M$ is moving to the to the left and thus the balancing of forces gives $m g+\mu M g=k X_{f}$ which gives the result in Equation (15) with the plus sign. Similarly, for the $n=$ even case, motion is from even $n$ to odd $(n+1)$ which means that $m$ is moving down and $M$ is moving to the right and thus the balancing forces gives $m g-\mu M g=k X_{f}$ which gives the result in Equation (15) with the negative sign.

It is interesting to find the value of $n$ at which the system reaches its final equilibrium state. This could be found by equating $x_{n}(n \pi)$ from Equation (9) with $X_{f}$ for the two cases $n=$ even and $n=$ odd which is given by Equation (15). Straightforward calculations give, for both cases

$$
n=\frac{1}{2}\left(\frac{m}{\mu M}-1\right)
$$

To find the total distance covered by either $m$ or $M$, one must notices that for the $n^{\text {th }}$ half-odd cycle $m$ is moving down while for the $n^{t h}$ half-even cycle it is moving up. Therefore, if we let $N$ to be the integer part of Equation (16), we have for $N=$ even,

$$
X_{\text {tot }}=2 \sum_{\mathrm{odd}}^{N-1} x_{n}-2 \sum_{\mathrm{even}}^{N} x_{n}+X_{f}
$$

The substitution of $x_{n}$ for $n=$ odd from Equation (12) and for $n=$ even from Equation (13) we get

$$
\begin{aligned}
X_{\text {tot }} & =\frac{4 g}{k}\left[m \sum_{\text {odd }}^{N-1} 1-\mu M \sum_{\text {odd }}^{N-1} n-\mu M \sum_{\text {even }}^{N} n\right]+X_{f} \\
& =\frac{4 g}{k}\left[m \frac{N}{2}-\mu M\left(\frac{N^{2}}{2}+\frac{N}{4}(N+2)\right)\right]+X_{f}
\end{aligned}
$$


Substituting for $X_{f}$ from Equation (15) with the negative sign, Equation (18) gives

$$
X_{\text {tot }}=\frac{2 g}{k} N[m-\mu M(N+1)]+\frac{g}{k}(m-\mu M) .
$$

It is interesting to note that the substitution $N=\frac{1}{2}\left(\frac{m}{\mu M}-1\right)$ enables us to write Equation (19) in terms of $N$ or in terms of the original quantities as

$$
X_{\text {tot }}=\frac{2 g}{k} \mu M N(N+1)=\frac{g}{2 k \mu M}\left(m^{2}-\mu^{2} M^{2}\right) .
$$

Similarly, the total distance for the case $N=$ odd, we have

$$
X_{\text {tot }}=2 \sum_{\text {odd }}^{N-2} x_{n}-2 \sum_{\text {even }}^{N-1} x_{n}+X_{f} .
$$

Noting that the two sums are similar to those in Equation (17) but with $N \rightarrow N-1$, so using this and substituting for $X_{f}$ from Equation (15) with the positive sign, we get

$$
X_{\text {tot }}=\frac{2 g}{k}(N-1)(m-\mu M)+\frac{g}{k} \mu M\left(\frac{m}{\mu M}+1\right),
$$

which upon using $\frac{m}{\mu M}=2 N+1$, the above equation can be written in terms of $N$ or in terms of the original quantities as

$$
X_{\text {tot }}=\frac{2 g}{k} \mu M N(N+1)=\frac{g}{2 k \mu M}\left(m^{2}-\mu^{2} M^{2}\right) .
$$

This is exactly the same as the total distance given by Equation (20) for the even $N$ case.

It is constructive to express the total distance $X_{t o t}$ in terms of the final position $X_{f}$. For $N=$ even, Equation (15) gives $X_{f}=\frac{g}{k}(m-\mu M)=\frac{g}{k} \mu M(2 N)$, so that $\frac{2 g}{k} \mu M=\frac{X_{f}}{N}$. Substituting this in Equation (20) gives

$$
X_{t o t}=(N+1) X_{f} .
$$

while for $N=$ odd, Equation (15) gives $X_{f}=\frac{g}{k}(m+\mu M)=\frac{2 g \mu M}{k}(N+1)$, so that $\frac{2 g}{k} \mu M=\frac{X_{f}}{N+1}$. Substituting this result into Equation (23) gives

$$
X_{\text {tot }}=N X_{f}
$$

\section{Energy Considerations}

Our result for the total distance covered by $m$ or $M$ can be checked from energy considerations. The gravitational potential energy of $m$ is consumed by energy stored in the spring and a work done against friction between $M$ and the surface. Namely, 


$$
m g X_{f}=\frac{1}{2} k X_{f}^{2}+\mu M g X_{t o t}
$$

Using Equation (15) for $X_{f}$, we get:

For $N=$ even, $X_{\text {tot }}=X_{f} \frac{1}{\mu M g}\left(m g-\frac{1}{2} g(m-\mu M)\right)=X_{f} \frac{1}{2}\left(\frac{m}{\mu M}+1\right)$ and using $\frac{m}{\mu M}=2 N+1$, we recover our result given in Equation (24), namely $X_{\text {tot }}=X_{f}(N+1)$.

For $N=$ odd, the substitution for $X_{f}$ from Equation (15) into Equation (26), we get $X_{\text {tot }}=X_{f} \frac{1}{\mu M g}\left(m g-\frac{1}{2} g(m+\mu M)\right)=X_{f} \frac{1}{2}\left(\frac{m}{\mu M}-1\right)$ and using $\frac{m}{\mu M}=2 N+1$, we recover our result given in Equation (24), namely, $X_{t o t}=N X_{f}$.

It is interesting to determine the energy stored in the spring, $U_{s}$ and the work done against friction $W_{f}$ and compare them with the initial gravitational potential energy, $U_{g}$ of the hanged mass $m$ relative to its final position.

Using Equation (15) for $X_{f}$ and Equation (20) for $X_{\text {tot }}$, we get

$$
\begin{gathered}
U_{g}=m g X_{f}=\frac{m g^{2}}{k}(m-\mu M), N=\text { even } \\
U_{g}=\frac{m g^{2}}{k}(m+\mu M), N=\text { odd } \\
U_{s}=\frac{1}{2} k X_{f}^{2}=\frac{g^{2}}{2 k}(m-\mu M)^{2}, N=\text { even } \\
=\frac{g^{2}}{2 k}(m+\mu M)^{2}, N=\text { odd } \\
W_{f}=\mu M g X_{\text {tot }}=\frac{g^{2}}{2 k}\left(m^{2}-\mu^{2} M^{2}\right), N=\text { even or odd }
\end{gathered}
$$

From the above equations, one can immediately find,

$$
\frac{U_{s}}{U_{g}}=\frac{1}{2} \frac{m \pm \mu M}{m}
$$

where the $-(+)$ sign is for $N=$ even (odd)

$$
\frac{W_{f}}{U_{g}}=\frac{1}{2} \frac{m \pm \mu M}{m}
$$

where the $+(-)$ sign is for $N=$ even (odd).

It is constructive and interesting to consider the special case when the horizontal surface is frictionless $(\mu \rightarrow 0)$ : Equations (27)-(29) give

$$
U_{g}=\frac{m^{2} g^{2}}{k}, U_{s}=W_{f}=\frac{m^{2} g^{2}}{2 k},
$$

and therefore,

$$
\frac{U_{s}}{U_{g}}=\frac{W_{f}}{U_{g}}=\frac{1}{2} .
$$


So we observe that even when the system is non-dissipative, half the initial gravitational potential energy will be stored in the spring while the other half is lost.

\section{Conclusion}

In this paper, we examined the dynamics of a classical system during its transition from a non-equilibrium state to a final equilibrium one. The position of the system at the end of the $n^{\text {th }}$-half cycle was calculated. The even and odd half cycle was examined and our results for the final position are consistent with force balancing for each parity of $n$. The number of half cycles is determined by the masses of the connected blocks and the coefficient of kinetic friction. Furthermore, the total distance covered by the system was determined. The energy involved during the system's transition was calculated for the two parity cases of n. Our results show that in the limit of vanishing coefficient of friction the energy stored in the spring is exactly half the initial gravitational potential energy and the other half is an energy loss. This is in complete analogy with the energy loss in the two-capacitor problem.

\section{Conflicts of Interest}

The author declares no conflicts of interest regarding the publication of this paper.

\section{References}

[1] Cohen, E.G.D. (2015) On the Transition of a Non-Equilibrium System to an Equilibrium State. The European Physical Journal Special Topics, 224, 801-807. https://doi.org/10.1140/epjst/e2015-02428-5

[2] Berthier, L. and Kurcham, J. (2013) Non-Equilibrium Glass Transitions in Driven and Active Matter. Nature Physics, 9, 310-314. https://doi.org/10.1038/nphys2592

[3] Fenf, H., Zhang, K. and Wang, J. (2014) Non-Equilibrium Transition State Rate Theory. Chemical Physics, 5, 3761.

[4] Nicolis, G. and Drigogine, I. (1971) Fluctuations in Non-Equilibrium Systems. Proceedings of the National Academy of Sciences of the United States of America, 68, 2102-2107. https://doi.org/10.1073/pnas.68.9.2102

[5] Zia, R.K.P., Shaw, L.B., Schmittmann, B. and Astalos, R.J. (2000) Contrasts between Equilibrium and Non-Equilibrium Steady States: Computer Aided Discoveries in Simple Lattice Asses. Computer Physics Communications, 127, 23-31. https://doi.org/10.1016/S0010-4655(00)00022-9

[6] Derrida, B. (2007) Non-Equilibrium Steady States: Fluctuations and Large Deviations of the Density and of the Current. Journal of Statistical Mechanics Theory and Experiment, 2007, Article ID: 07023. https://doi.org/10.1088/1742-5468/2007/07/P07023

[7] Odagaki, T. (2017) Non-Equilibrium Statistical Mechanics Based on the Free Energy Landscape and Its Applications to Glassy Systems. Journal of the Physical Society of Japan, 86, Article ID: 082001. https://doi.org/10.7566/JPSJ.86.082001

[8] Rastogi, R.P. and Srivastava, R.C. (2008) Causality Princiole, Non-Equilibrium Thermodynamics and Non-Linear Science of Open Systems. Journal of Scientific \& 
Industrial Research, 67, 747-758.

[9] Rabani, E. and Berne, B.J. (1998) Energy Dissipation in Non-Linear Systems Coupled to a Bath: On the Use of Perturbative Maps. Journal of Physical Chemistry $A, 102,9380-9389$. https://doi.org/10.1021/jp9814653

[10] Xiong, Y, Wang, T. and Teng, P. (2016) The Effect of Dissipation on Topological Mechanical Systems. Scientific Reports, 6, Article ID: 43572. https://doi.org/10.1038/srep32572

[11] Perieversev, A., Pereversev, Y. and Prezhodo, O.V. (208) Dissipation of Classical Energy in Non-Linear Quantum Systems. Journal of Chemical Physics, 128, Article ID: 134107.

[12] Ghadirian, R., Stait-Gardner, T., Hennessy, A. and Price, W.S. (2011) Energy Dissipation in Porous Media for Equilibrium and Non-Equilibrium Translational Motion. Journal of the Basic Principles of Diffusion Theory Experiment and Applications, 15, 1-21.

[13] Bonanca, M.V.S. and de Aquiar, M.A.M. (2006) Equilibrium via Interaction with Chaotic Systems. Physica A, 365, 333-340.

https://doi.org/10.1016/j.physa.2005.09.062

[14] AL-Jaber, S.M. and Salih, S.K. (2000) Energy Considerations in the Two-Capacitor Problem. European Journal of Physics, 21, 341. https://doi.org/10.1088/0143-0807/21/4/307

[15] Mita, K. and Boufaida, M. (1999) Ideal Capacitor Circuits and Energy Conservation. American Journal of Physics, 67, 737-738. https://doi.org/10.1119/1.19363

[16] Choy, T.C. (2004) Capacitors Can Radiate: Further Results for the Two-Capacitor Problem. American Journal of Physics, 72, 662-670. https://doi.org/10.1119/1.1643371

[17] Timothy, B.B., Hite, D. and Singh, N. (2002) The Two-Capacitor Problem with Radiation. American Journal of Physics, 70, 415-420. https://doi.org/10.1119/1.1435344

[18] Abu-Labdeh, A.M. and AL-Jaber, S.M. (2008) Energy Consideration from Non-Equilibrium to Equilibrium State in the Process of Charging a Capacitor. Journal of Electrostatics, 66, 190-192. https://doi.org/10.1016/j.elstat.2007.12.002

[19] AL-Jaber, S.M. and Abu-Labdeh, A.M. (2011) Energy Consideration in the Process of Transition to Equilibrium State. Natural Science, 3, 136-140. https://doi.org/10.4236/ns.2011.32019

[20] Lee, K. (2009) The Two-Capacitor Problem Revisited: A Mechanical Harmonic Oscillator Model Approach. European Journal of Physics, 30, 69-74. https://doi.org/10.1088/0143-0807/30/1/007

[21] Lara, V.O.M., Lima, A.P. and Costa, A. (2015) Entropic Considerations in the Two-Capacitor Problem. Revista Brasilean de Ensino de Fisica, 37, 1301-1306. https://doi.org/10.1590/S1806-11173711650

[22] Libii, J.N. (2009) Demonstration of Energy Dissipation in a Spring-Mass System Undergoing Free Oscillations in Air. World Transactions on Engineering and Technology Education, 7, 28-33.

[23] Nijmeijera, H. (2004) Energy Dissipation of a Friction Damper. Journal of Sound and Vibration, 278, 539-561. https://doi.org/10.1016/j.jsv.2003.10.051

[24] Triana, C.A. and Fajardo, F. (2013) Experimental Study of a Simple Harmonic Motion of a Spring-Mass System as a Function of Spring Diameter. Revista Brasilean de Ensino de Fisica, 35, 43051-43058. 
[25] Krim, J. (2012) Friction and Energy Dissipation Mechanisms in Adsorbed Molecules and Molecularly Thin Films. Advances in Physics, 61, 155-323. https://doi.org/10.1080/00018732.2012.706401

[26] Onorato, P., Mascoli, D. and Deambrosis, A. (2010) Damped Oscillations and Equilibrium in a Spring-Mass System Subjected to Sliding Friction Forces: Integrating Experimental and Theoretical Analysis. American Journal of Physics, 78, 1120-1126. https://doi.org/10.1119/1.3471936

[27] Besson, U., Borghi, L., De Ambrosis and Mascheretti, P. (2007) How to Teach Friction: Experiments and Models. American Journal of Physics, 75, 1106-1113. https://doi.org/10.1119/1.2779881

[28] Molina, M.I. (2004) Exponential versus Linear Amplitude Decay in Damped Oscillators. Physics Teacher, 42, 485-487. https://doi.org/10.1119/1.1814324

[29] Kamela, M. (2007) An Oscillating System with Sliding Friction. Physics Teacher, 45, 110-113. https://doi.org/10.1119/1.2432089 